MATHEMATICS OF COMPUTATION

VOLUME 57, NUMBER 195

JULY 1991, PAGES 109-122

\title{
PSEUDOSPECTRAL METHOD FOR THE “GOOD” BOUSSINESQ EQUATION
}

\author{
J. DE FRUTOS, T. ORTEGA, AND J. M. SANZ-SERNA
}

\begin{abstract}
We prove the nonlinear stability and convergence of a fully discrete, pseudospectral scheme for the "good" Boussinesq equation $u_{t}=-u_{x x x x}+$ $u_{x x}+\left(u^{2}\right)_{x x}$. Numerical comparisons with finite difference schemes are also reported.
\end{abstract}

\section{INTRODUCTION}

The "good" Boussinesq (GB) equation

$$
u_{t t}=-u_{x x x x}+u_{x x}+\left(u^{2}\right)_{x x}
$$

is similar to the well-known Korteweg-de Vries (KdV) and cubic Schrödinger (CS) equation in that it provides a balance between dispersion and nonlinearity that leads to the existence of solitons. However, the GB equation possesses some remarkable peculiarities. For instance, solitary waves (i) only exist for a finite range of velocities, (ii) can merge into a single solitary wave, (iii) can interact to give rise to so-called anti-solitons [10].

The numerical and analytical study of the GB equation is only beginning. Two recent papers are $[9,10]$. The first of these articles provides an exact formula for the interaction of solitons. The second is devoted to an analysis of the soliton interaction mechanism and to the existence and regularity of solutions of the initial-value problem. While numerical experiments are reported in [9], little analysis is given there of the stability and convergence of the methods employed.

In [11] two of the present authors have shown the nonlinear stability and convergence of a family of finite difference schemes for the numerical solution of the GB equation. While these schemes may provide a useful integration method when high accuracy is not required, finite difference algorithms are often (see, e.g., $[15,17])$ judged not to be competitive with their spectral and pseudospectral counterparts. The aim of the present work is the analysis and assessment of a pseudospectral time-discrete method for the GB equation. General background references are $[5,1,6,16]$.

Received January 12, 1989.

1980 Mathematics Subject Classification (1985 Revision). Primary 65M10. 
Throughout the paper, we consider that, at each time level, the algorithms provide a vector $\mathbf{U}$ of approximations to nodal values of the theoretical solution $u$. Hence, errors are measured by means of discrete norms. In an alternative point of view, the algorithms could have been seen as providing, at each time level, a trigonometric polynomial that approximates $u$ over the spatial domain. It would not be difficult to translate the discrete-norm bounds obtained in this paper into continuous-norm bounds for the difference between $u$ and the trigonometric interpolant of the discrete solution $\mathbf{U}$.

Our estimates for the global error are derived via the "stability plus consistency" approach. The main problem encountered lies in the stability study of the aliasing error in the nonlinear term. To alleviate these difficulties, the analysis is carried out after twice integrating the differential equation with respect to $x$, so as to remove the derivatives occurring in the nonlinear term. This is roughly equivalent to the use of negative Sobolev norms (see the remark after Theorem 4.2).

The paper is organized as follows. In $\S 2$ we present the problem to be solved and the numerical method. In $\S 3$ we study the energy norm, which is the key ingredient in the stability and convergence analysis of $\S 4$. Finally, $\hat{\S} 5$ is devoted to numerical experiments.

\section{A NUMERICAL METHOD}

We consider the periodic problem

$$
\begin{gathered}
u_{t t}=-u_{x x x x}+u_{x x}+\left(u^{2}\right)_{x x}, \quad-\infty<x<\infty, 0 \leq t \leq T<\infty, \\
u(x, t)=u(x+1, t), \quad-\infty<x<\infty, 0 \leq t \leq T, \\
u(x, 0)=u^{0}(x), \quad-\infty<x<\infty, \\
u_{t}(x, 0)=v^{0}(x), \quad-\infty<x<\infty,
\end{gathered}
$$

where the data $u^{0}, v^{0}$ are 1-periodic functions, which are assumed to be smooth enough for (2.1)-(2.4) to have a unique solution, classical or generalized (see [10]). While, for simplicity, we have chosen the period in (2.2) to be 1 , it is clear that what follows can be readily extended to cover the case of an arbitrary period.

We now define our numerical method. If $J$ is a positive integer, we set $h=1 /(2 J)$ and consider the mesh points $x_{j}=j h, j$ is an integer. We denote by $\mathbb{Z}_{h}$ the space of real, 1-periodic functions defined on the mesh. Thus each element $\mathbf{V} \in \mathbb{Z}_{h}$ is a sequence $\left\{V_{j}\right\}_{j=0, \pm 1, \ldots}$ with $V_{j}=V_{j+2 J}, j=0, \pm 1, \ldots$. The notation $[\mathbf{V}]_{p}^{-}$refers to the $p$ th discrete Fourier coefficient of $\mathbf{V}$, i.e.,

$$
[\mathbf{V}]_{p}^{-}=\frac{1}{2 J} \sum_{0 \leq j \leq 2 J}^{\prime \prime} V_{j} \exp (-2 \pi i p j h), \quad-J \leq p \leq J,
$$


where the double prime in the summation means that the first and last terms are halved. The recovery of $\mathbf{V}$ from its Fourier coefficients is achieved by the inverse discrete Fourier transorm

$$
V_{j}=V^{*}\left(x_{j}\right), \quad j=0, \pm 1, \ldots,
$$

where $V^{*}(x)$ is the trigonometric interpolant of $\mathbf{V}$ given by

$$
V^{*}(x)=\sum_{-J \leq p \leq J}^{\prime \prime}[\mathbf{V}]_{p}^{-} \exp (2 \pi i p x), \quad-\infty<x<\infty .
$$

Differentiation of (2.5) with respect to $x$ and evaluation at the mesh points lead to the following definition of the standard second difference pseudospectral operator $D^{2}$ mapping $\mathbb{Z}_{h}$ into itself:

$$
\begin{aligned}
\left(D^{2} \mathbf{V}\right)_{j}=\sum_{-J \leq p \leq J}^{\prime \prime}[\mathbf{V}]_{p}(2 \pi i p)^{2} \exp (2 \pi i p j h), & \\
& \mathbf{V} \in \mathbb{Z}_{h}, j=0, \pm 1, \ldots .
\end{aligned}
$$

Of course, equality (2.6) can be written as the following simple formula for the Fourier coefficients:

$$
\left[D^{2} \mathbf{V}\right]_{p}^{-}=(2 \pi i p)^{2}[\mathbf{V}]_{p}^{\hat{n}}, \quad-J \leq p \leq J .
$$

The operator $D^{4}$ in $\mathbb{Z}_{h}$ is, by definition, the composition $D^{2} D^{2}$.

Next, let $k$ denote a parameter $0<k<T$ and consider the time levels $t_{n}=n k, n=0,1, \ldots, N$, with $N=[T / k]$. In the sequel a superscript $n$ denotes a quantity associated with time level $t_{n}$. With these notations, we consider the pseudospectral scheme

$$
\begin{aligned}
& \left(\mathbf{U}^{n+1}-2 \mathbf{U}^{n}+\mathbf{U}^{n-1}\right) / k^{2} \\
& =-\frac{1}{4}\left\{D^{4} \mathbf{U}^{n+1}+2 D^{4} \mathbf{U}^{n}+D^{4} \mathbf{U}^{n-1}\right\}+D^{2} \mathbf{U}^{n}+D^{2}\left(\mathbf{U}^{n}\right)^{2}, \\
& n=0,1, \ldots, N-1,
\end{aligned}
$$

with initial values

$$
\begin{gathered}
\mathbf{U}^{0}=\boldsymbol{\alpha}, \\
\left(\mathbf{U}^{1}-\mathbf{U}^{0}\right) / k=\boldsymbol{\beta},
\end{gathered}
$$

where $\boldsymbol{\alpha}, \boldsymbol{\beta} \in \mathbb{Z}_{h}$ are given approximations to $\mathbf{r}_{h} u^{0}, \mathbf{r}_{h} v^{0}$, the grid restrictions of the functions $u^{0}$ and $v^{0}$ in (2.3)-(2.4). The elements $\mathbf{U}^{n}$ are, of course, meant to approximate the grid restriction $\mathbf{u}^{n}=\mathbf{r}_{h} u^{n}$, where $u^{n}=u\left(\cdot, t_{n}\right)$.

For implementation purposes, it is best to Fourier-transform (2.8) to get, after taking (2.7) into account,

$$
\begin{aligned}
& \left(\left[\mathbf{U}^{n+1}\right]_{p}-2\left[\mathbf{U}^{n}\right]_{p}+\left[\mathbf{U}^{n-1}\right]_{p}\right) / k^{2}
\end{aligned}
$$

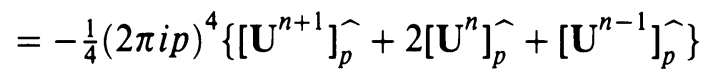

$$
\begin{aligned}
& +(2 \pi i p)^{2}\left\{\left[\mathbf{U}^{n}\right]_{p}^{\hat{p}}+\left[\left(\mathbf{U}^{n}\right)^{2}\right]_{p}^{\hat{p}}\right\}, \quad-J \leq p \leq J \text {. }
\end{aligned}
$$


The last equations, for each $p$, give $\left[\mathbf{U}^{n+1}\right]_{p}^{\wedge}$ in terms of $\left[\mathbf{U}^{n}\right]_{p}^{-},\left[\mathbf{U}^{n-1}\right]_{p}^{-}$, and $\left[\left(\mathbf{U}^{n}\right)^{2}\right]_{p}^{-}$. Therefore, if $\left[\mathbf{U}^{n}\right]_{p}^{-},\left[\mathbf{U}^{n-1}\right]_{p}^{-}$are in storage, the computation of the solution $\mathbf{U}^{n+1}$ requires the computation of the Fourier transform of $\left(\mathbf{U}^{n}\right)^{2}$, plus the computation of an inverse Fourier transform to recover $\mathbf{U}^{n+1}$ from its Fourier coefficients. Of course, in practice, $2 J$ is chosen to be of the form $2^{m}$, $m$ an integer, and the transforms are performed by FFT techniques [2].

The weights $1 / 4,1 / 2,1 / 4$ of $D^{4} \mathbf{U}^{n+1}, D^{4} \mathbf{U}^{n}, D^{4} \mathbf{U}^{n-1}$ in (2.8) have been chosen for well-known accuracy reasons, but clearly other choices of weights are possible and can be analyzed by using the techniques below (cf. [11]). Also note that the time-continuous version of $(2.8)$ is appealing when combined with the use of a standard ODE package. The analysis of such a time-continuous algorithm can be done along the lines of the study presented in this paper of the fully-discrete method (2.8).

\section{THE ENERGY NORM}

The convergence analysis to be carried out in the next section is based on the use of an appropriate energy norm for the discrete solution $\mathbf{U}^{n}$. In order to motivate our choice of energy norm, it is useful to consider the linear problem given by the principal part of (2.1),

$$
u_{t t}=-u_{x x x x}, \quad-\infty<x<\infty, 0 \leq t \leq T<\infty,
$$

along with (2.2)-(2.4).

We denote by $L^{2}$ the usual space of square-integrable 1-periodic functions, endowed with the standard norm $\|\cdot\|$ and inner product $(\cdot, \cdot)$. Furthermore, we denote by $\partial_{x}^{2}$ the operator $d^{2} / d x^{2}$ and by $l$ the average functional

$$
l(v)=\int_{0}^{1} v(x) d x .
$$

If $v$ is a function in $L^{2}$, Fourier analysis easily shows that there is a unique function $\partial_{x}^{-2} v \in L^{2}$ such that

$$
\partial_{x}^{2}\left(\partial_{x}^{-2} v\right)=v-l(v), \quad l\left(\partial_{x}^{-2} v\right)=l(v) .
$$

We apply the operator $\partial_{x}^{-2}$ to $(3.1)$ and take the inner product with $\partial_{x}^{-2} u_{t}$ to get

$$
\left(\partial_{x}^{-2} u_{t t}, \partial_{x}^{-2} u_{t}\right)=-\left(\partial_{x}^{2} u, \partial_{x}^{-2} u_{t}\right)
$$

Integration by parts yields

$$
\left(\partial_{x}^{-2} u_{t t}, \partial_{x}^{-2} u_{t}\right)+\left(u, u_{t}\right)=\left(u, l\left(u_{t}\right)\right)
$$

or

$$
\begin{aligned}
\frac{1}{2} \frac{d}{d t}\left\{\left\|\partial_{x}^{-2} u_{t}\right\|^{2}+\|u\|^{2}\right\} & =l(u) l\left(u_{t}\right)=l(u) l\left(\partial_{x}^{-2} u_{t}\right) \\
& \leq \frac{1}{2}\left\{\left\|\partial_{x}^{-2} u_{t}\right\|^{2}+\|u\|^{2}\right\} .
\end{aligned}
$$


Now the Gronwall lemma leads to an estimate

$$
\left\|\partial_{x}^{-2} u_{t}\right\|^{2}+\|u\|^{2} \leq C\left\{\left\|\partial_{x}^{-2} v^{0}\right\|^{2}+\left\|u^{0}\right\|^{2}\right\}, \quad 0 \leq t \leq T,
$$

for the solutions of the problem (3.1), (2.2)-(2.4). This shows that the problem is well posed in the following energy norm for pairs $(v, u) \in L^{2} \times L^{2}$ :

$$
\|(v, u)\|_{E}=\left(\left\|\partial_{x}^{-2} v\right\|^{2}+\|u\|^{2}\right)^{1 / 2}
$$

To construct a discrete analogue of (3.3), we begin by defining the operator $D^{-2}$ in $\mathbb{Z}_{h}$ such that (cf. (3.2)),

$$
D^{2}\left(D^{-2}(\mathbf{V})\right)=\mathbf{V}-[\mathbf{V}]_{0} \mathbf{1}, \quad\left[D^{-2} \mathbf{V}\right]_{0}=[\mathbf{V}]_{0} .
$$

Here and later, 1 represents the grid function that takes the value 1 at every grid point. Fourier analysis reveals once more that (3.4) uniquely defines $D^{-2} \mathbf{V}$. Namely, the Fourier coefficients of $D^{-2} \mathbf{V}$ are defined by

$$
\left[D^{-2} \mathbf{V}\right]_{p}^{-}=(2 \pi i p)^{-2}[\mathbf{V}]_{p}^{\widehat{n}}, \quad p= \pm 1, \pm 2, \ldots, \pm J,
$$

and the second condition in (3.4). This Fourier representation also shows that $D^{-2}$ commutes with $D^{2}$.

Next, if $\left(\mathbf{V}, \mathbf{V}^{*}\right) \in \mathbb{Z}_{h} \times \mathbb{Z}_{h}$, we set

$$
Q_{k}\left(\mathbf{V}, \mathbf{V}^{*}\right)=\left\|D^{-2}\left(\mathbf{V}-\mathbf{V}^{*}\right) / k\right\|^{2}+\frac{1}{4}\left(\|\mathbf{V}\|^{2}+2\left(\mathbf{V}, \mathbf{V}^{*}\right)+\left\|\mathbf{V}^{*}\right\|^{2}\right)
$$

where $\|\cdot\|$ denotes the standard discrete $L^{2}$-norm

$$
\|\mathbf{V}\|^{2}=\sum_{0 \leq j \leq 2 J}^{\prime \prime} h V_{j}^{2}
$$

and $(\cdot, \cdot)$ represents the corresponding inner product. (Note that the same symbols $(\cdot, \cdot),\|\cdot\|$ are used for the continuous and discrete cases, but no confusion is possible.) The operators $D^{2}, D^{4}, D^{-2}$ are clearly selfadjoint with respect to $(\cdot, \cdot)$.

Proposition. For $\left(\mathbf{V}, \mathbf{V}^{*}\right) \in \mathbb{Z}_{h} \times \mathbb{Z}_{h}$ we have

$$
\begin{aligned}
Q_{k}\left(\mathbf{V}, \mathbf{V}^{*}\right) & \leq\left\|D^{-2}\left(\mathbf{V}-\mathbf{V}^{*}\right) / k\right\|^{2}+\frac{1}{2}\left(\|\mathbf{V}\|^{2}+\frac{1}{2}\left\|\mathbf{V}^{*}\right\|^{2}\right) \\
& \leq K(r) Q_{k}\left(\mathbf{V}, \mathbf{V}^{*}\right)
\end{aligned}
$$

where $K(r)=\left(4+\pi^{4} r^{2}\right) / 4$, with $r$ the mesh ratio $r=k / h^{2}$.

Proof. We employ the technique of the proof of Proposition 3.1 of [11]. The first inequality in (3.7) is obvious. To prove the second, we introduce the following quadratic form $P_{k}$ in $\mathbb{Z}_{h} \times \mathbb{Z}_{h}$ :

$$
P_{k}\left(\mathbf{V}, \mathbf{V}^{*}\right)=\left\|D^{-2}\left(\mathbf{V}-\mathbf{V}^{*}\right) / k\right\|^{2}+\frac{1}{2}\|\mathbf{V}\|^{2}+\frac{1}{2}\left\|\mathbf{V}^{*}\right\|^{2}
$$

and compare the eigenvalues/functions of $P_{k}$ and $Q_{k}$. 
From (3.6), the selfadjoint operator in $\mathbb{Z}_{h} \times \mathbb{Z}_{h}$ associated with $Q_{k}$ may be written in block form as

$$
\left[\begin{array}{cc}
k^{-2} D^{-4}+\frac{1}{4} I & -k^{-2} D^{-4}+\frac{1}{4} I \\
-k^{-2} D^{-4}+\frac{1}{4} I & k^{-2} D^{-4}+\frac{1}{4} I
\end{array}\right]
$$

where $D^{-4}$ is the composition $D^{-2} D^{-2}$. Therefore, if $\left(\mathbf{V}, \mathbf{V}^{*}\right)$ is an eigenfunction associated with the eigenvalue $\lambda$, then

$$
\begin{aligned}
k^{-2} D^{-4} \mathbf{V}+\frac{1}{4} \mathbf{V}-k^{-2} D^{-4} \mathbf{V}^{*}+\frac{1}{4} \mathbf{V}^{*} & =\lambda \mathbf{V}, \\
-k^{-2} D^{-4} \mathbf{V}+\frac{1}{4} \mathbf{V}+k^{-2} D^{-4} \mathbf{V}^{*}+\frac{1}{4} \mathbf{V}^{*} & =\lambda \mathbf{V}^{*}
\end{aligned}
$$

By adding and subtracting these equations we obtain

$$
\begin{gathered}
\frac{1}{2}\left(\mathbf{V}+\mathbf{V}^{*}\right)=\lambda\left(\mathbf{V}+\mathbf{V}^{*}\right), \\
k^{-2} D^{-4}\left(\mathbf{V}-\mathbf{V}^{*}\right)=\lambda\left(\mathbf{V}-\mathbf{V}^{*}\right) .
\end{gathered}
$$

When eigenfunctions with $\mathbf{V}=\mathbf{V}^{*}$ are looked for, (3.9) holds and (3.8) reveals that $\lambda=1 / 2$ is an eigenvalue with multiplicity $\operatorname{dim}\left(\mathbb{Z}_{h}\right)=2 J$. On the other hand, if eigenfunctions with $\mathbf{V}=-\mathbf{V}^{*}$ are sought, (3.8) holds and (3.9) implies that $\lambda$ is an eigenvalue of $2 k^{-2} D^{-4}$ and $\mathbf{V}$ the associated eigenfunction.

Turning now to $P_{k}$, a similar argument yields that the eigenvalues/functions of $P_{k}$ are, on the one hand, $\left\{1 / 2,\left(\mathbf{V}, \mathbf{V}^{*}\right)\right\}, \mathbf{V}$ arbitrary, and, on the other hand, $\left\{1 / 2+\mu,\left(\mathbf{V},-\mathbf{V}^{*}\right)\right\}$, with $\mu \in \operatorname{Spec}\left(2 k^{-2} D^{-4}\right)$ and $\mathbf{V}$ the associated eigenfunction. Since $P_{k}, Q_{k}$ possess a common set of eigenfunctions, the second inequality in (3.7) is equivalent to the condition

$$
\lambda\left(P_{k}\right) \leq\left[\left(4+\pi^{4} r^{2}\right) / 4\right] \lambda\left(Q_{k}\right)
$$

for the corresponding eigenvalues, i.e.,

$$
\mu+1 / 2 \leq\left[\left(4+\pi^{4} r^{2}\right) / 4\right] \mu, \quad \mu \in \operatorname{Spec}\left(2 k^{-2} D^{-4}\right) .
$$

Now (3.10) is easily seen to hold, after noticing that (3.4)-(3.5) imply that the eigenvalues of $D^{-2}$ are 1 and $1 /(-2 \pi p)^{2}, p= \pm 1, \pm 2, \ldots, \pm(J-1), J$.

As a first consequence of the proposition, note that $Q_{k}$ is positive definite and that it is therefore possible to define a discrete energy norm by

$$
\left\|\left(\mathbf{V}, \mathbf{V}^{*}\right)\right\|_{E}=Q_{k}\left(\mathbf{V}, \mathbf{V}^{*}\right)^{1 / 2}, \quad\left(\mathbf{V}, \mathbf{V}^{*}\right) \in \mathbb{Z}_{h} \times \mathbb{Z}_{h}
$$

Furthermore, note that (3.6) implies that the energy norm is equivalent to the Sobolev norm (cf. (3.3))

$$
\left\{\left\|D^{-2}\left(\mathbf{V}-\mathbf{V}^{*}\right) / k\right\|^{2}+\frac{1}{2}\|\mathbf{V}\|^{2}+\frac{1}{2}\left\|\mathbf{V}^{*}\right\|^{2}\right\}^{1 / 2} .
$$

The equivalence is uniform in $k, h$, provided that the grids are refined subject to a restriction

$$
r_{\max }:=\sup \left(k / h^{2}\right)<\infty
$$




\section{NONLINEAR STABILITY AND CONVERGENCE}

We now investigate the nonlinear stability of the algorithm (2.8). To this end, let $\mathbf{V}^{0}, \mathbf{v}^{1}, \ldots, \mathbf{V}^{N}$ and $\mathbf{w}^{0}, \mathbf{w}^{1}, \ldots, \mathbf{w}^{N}$ be two sequences of elements $\mathbf{V}^{n}$, $\mathbf{W}^{n} \in \mathbb{Z}_{h}, n=0,1, \ldots, N$, and define the residuals

$$
\begin{aligned}
\mathbf{F}^{0}= & \mathbf{V}^{0}-\boldsymbol{\alpha} \\
\mathbf{F}^{1}= & \mathbf{V}^{1}-\boldsymbol{\alpha}-k \boldsymbol{\beta}, \\
\mathbf{F}^{n+1}= & k^{-2}\left(\mathbf{V}^{n+1}-2 \mathbf{V}^{n}+\mathbf{V}^{n-1}\right)+\frac{1}{4}\left\{D^{4} \mathbf{V}^{n+1}+2 D^{4} \mathbf{V}^{n}+D^{4} \mathbf{V}^{n-1}\right\} \\
& -D^{2} \mathbf{V}^{n}-D^{2}\left(\mathbf{V}^{n}\right)^{2}, \quad n=1,2, \ldots, N-1,
\end{aligned}
$$

and

$$
\begin{aligned}
\mathbf{G}^{0}= & \mathbf{W}^{0}-\boldsymbol{\alpha}, \\
\mathbf{G}^{1}= & \mathbf{W}^{1}-\boldsymbol{\alpha}-k \boldsymbol{\beta}, \\
\mathbf{G}^{n+1}= & k^{-2}\left(\mathbf{W}^{n+1}-2 \mathbf{W}^{n}+\mathbf{W}^{n-1}\right)+\frac{1}{4}\left\{D^{4} \mathbf{W}^{n+1}+2 D^{4} \mathbf{W}^{n}+D^{2} \mathbf{W}^{n-1}\right\} \\
& -D^{2} \mathbf{W}^{n}-D^{2}\left(\mathbf{W}^{n}\right)^{2}, \quad n=1,2, \ldots, N-1 .
\end{aligned}
$$

Thus, $\left\{\mathbf{V}^{n}\right\},\left\{\mathbf{W}^{n}\right\}$ can be viewed as perturbed solutions of $(2.8)$ with $\left\{\mathbf{F}^{n}\right\}$, $\left\{\mathbf{G}^{n}\right\}$ the corresponding perturbations. The stability analysis consists of estimating the size of the differences $\mathbf{V}^{n}-\mathbf{W}^{n}$ in terms of the size of the differences $\mathbf{F}^{n}-\mathbf{G}^{n}$.

Theorem 4.1. Assume that (i) the grids are refined according to (3.12) and (ii) the, possibly generalized, solution $u$ of $(2.1)-(2.4)$ is a bounded function on $0 \leq x \leq 1,0 \leq t \leq T$. Let $\mu$ be an arbitrary positive number, and set

$$
M=\max \{u: 0 \leq x \leq 1,0 \leq t \leq T\} .
$$

Under these hypotheses, there exist a constant $k_{0}$, depending only on $r_{\max }$ and a constant $S$, depending on $r_{\max }, \mu$, and $M$, such that if $\left\{\mathbf{V}^{n}\right\},\left\{\mathbf{W}^{n}\right\},\left\{\mathbf{F}^{n}\right\}$, $\left\{\mathbf{G}^{n}\right\}$ are as above and

$$
\begin{aligned}
& \max _{0 \leq n \leq N-1}\left\|\left(\mathbf{V}^{n+1}-\mathbf{u}^{n+1}, \mathbf{V}^{n}-\mathbf{u}^{n}\right)\right\|_{E} \leq \mu h^{1 / 2}, \\
& \max _{0 \leq n \leq N-1}\left\|\left(\mathbf{W}^{n+1}-\mathbf{u}^{n+1}, \mathbf{W}^{n}-\mathbf{u}^{n}\right)\right\|_{E} \leq \mu h^{1 / 2},
\end{aligned}
$$

then, for $k<k_{0}$,

$$
\begin{aligned}
& \max _{0 \leq n \leq N-1}\left\|\left(\mathbf{V}^{n+1}-\mathbf{W}^{n+1}, \mathbf{V}^{n}-\mathbf{W}^{n}\right)\right\|_{E} \\
& \left.\quad \leq \exp (S T)\left\{\| \mathbf{F}^{1}-\mathbf{G}^{1}, \mathbf{F}^{0}-\mathbf{G}^{0}\right)\left\|_{E}+\sum_{2 \leq n \leq N} k\right\| D^{-2}\left(\mathbf{F}^{n}-\mathbf{G}^{n}\right) \|\right\} .
\end{aligned}
$$

Proof. With the abbreviations $\mathbf{e}^{n}=\mathbf{V}^{n}-\mathbf{W}^{n}, \mathbf{l}^{n}=\mathbf{F}^{n}-\mathbf{G}^{n}, n=0,1, \ldots, N$, after subtracting (4.1) and (4.2), we get, for $n=1,2, \ldots, N-1$,

$$
\begin{gathered}
k^{-2}\left(\mathbf{e}^{n+1}-2 \mathbf{e}^{n}+\mathbf{e}^{n-1}\right)+\frac{1}{4}\left\{D^{4} \mathbf{e}^{n+1}+2 D^{4} \mathbf{e}^{n}+D^{4} \mathbf{e}^{n-1}\right\} \\
-D^{2} \mathbf{e}^{n}-D^{2}\left[\left(\mathbf{V}^{n}\right)^{2}-\left(\mathbf{W}^{n}\right)^{2}\right]=\mathbf{l}^{n+1} .
\end{gathered}
$$


Application of the operator $D^{-2}$ to the previous equality and some rearrangement lead to

$$
\begin{aligned}
& k^{-2}\left(D^{-2} \mathbf{e}^{n+1}-2 D^{-2} \mathbf{e}^{n}+D^{-2} \mathbf{e}^{n-1}\right)+\frac{1}{4}\left\{D^{2} \mathbf{e}^{n+1}+2 D^{2} \mathbf{e}^{n}+D^{2} \mathbf{e}^{n-1}\right\} \\
&= \mathbf{e}^{n}-\left[\mathbf{e}^{n}\right]_{0}^{-} \mathbf{1}+\left(\mathbf{V}^{n}\right)^{2}-\left(\mathbf{W}^{n}\right)^{2} \\
&-\left[\left(\mathbf{V}^{n}\right)^{2}-\left(\mathbf{W}^{n}\right)^{2}\right]_{0}^{-} \mathbf{1}-D^{-2} \mathbf{l}^{n+1}, \quad n=1,2, \ldots, N-1 .
\end{aligned}
$$

We now take the inner product of (4.6) and $D^{-2}\left(\mathbf{e}^{n+1}-\mathbf{e}^{n}\right)+D^{-2}\left(\mathbf{e}^{n}-\mathbf{e}^{n-1}\right)$, and in the result rearrange the left-hand side and apply Cauchy-Schwarz to the right-hand side. This results in

$$
\begin{aligned}
& Q_{k}\left(\mathbf{e}^{n+1}, \mathbf{e}^{n}\right)-Q_{k}\left(\mathbf{e}^{n}, \mathbf{e}^{n-1}\right)+\frac{1}{4}\left\{\left[\mathbf{e}^{n+1}\right]_{0}^{\hat{0}}+2\left[\mathbf{e}^{n}\right]_{0}+\left[\mathbf{e}^{n-1}\right]_{0}^{-}\right\} \\
& +\left\{\left[D^{-2}\left(\mathbf{e}^{n+1}-\mathbf{e}^{n}\right)\right]_{0}^{\hat{0}}+\left[D^{-2}\left(\mathbf{e}^{n}-\mathbf{e}^{n-1}\right)\right]_{0}\right\} \\
& \leq\left\|\mathbf{e}^{n}-\left[\mathbf{e}^{n}\right]_{0}^{\hat{1}} \mathbf{1}+\left(\mathbf{V}^{n}\right)^{2}-\left(\mathbf{W}^{n}\right)^{2}-\left[\left(\mathbf{V}^{n}\right)^{2}-\left(\mathbf{W}^{n}\right)^{2}\right]_{0}^{\hat{1}} \mathbf{1}-D^{-2} \mathbf{l}^{n+1}\right\| \\
& +\left(\left\|D^{-2}\left(\mathbf{e}^{n+1}-\mathbf{e}^{n}\right)\right\|+\left\|D^{-2}\left(\mathbf{e}^{n}-\mathbf{e}^{n-1}\right)\right\|\right), \quad n=1,2, \ldots, N-1 \text {. }
\end{aligned}
$$

Now the definition of $Q_{k}$ implies

$$
\left\|D^{-2}\left(\mathbf{V}-\mathbf{V}^{*}\right)\right\| \leq k\left[Q_{k}\left(\mathbf{V}, \mathbf{V}^{*}\right)\right]^{1 / 2}, \quad \mathbf{V}, \mathbf{V}^{*} \in \mathbb{Z}_{h},
$$

and therefore (4.7) leads to

$$
\begin{aligned}
& \left\|\left(\mathbf{e}^{n+1}, \mathbf{e}^{n}\right)\right\|_{E}-\left\|\left(\mathbf{e}^{n}, \mathbf{e}^{n-1}\right)\right\|_{E} \\
& \leq k\left|\frac{1}{4}\left\{\left[\mathbf{e}^{n+1}\right]_{0}^{-}+2\left[\mathbf{e}^{n}\right]_{0}^{-}+\left[\mathbf{e}^{n-1}\right]_{0}^{-}\right\}\right| \\
& +k \| \mathbf{e}^{n}-\left[\mathbf{e}^{n}\right]_{0}^{\hat{1}}+\left(\mathbf{V}^{n}\right)^{2}-\left(\mathbf{W}^{n}\right)^{2} \\
& -\left[\left(\mathbf{V}^{n}\right)^{2}-\left(\mathbf{W}^{n}\right)^{2}\right]_{0}^{\hat{1}}+D^{-2} \mathbf{l}^{n+1} \|, \\
& n=1,2, \ldots, N-1 .
\end{aligned}
$$

The proposition of the previous section shows that

$$
\begin{aligned}
\frac{1}{4}\left|\left[\mathbf{e}^{n+1}\right]_{0}^{-}+\left[\mathbf{e}^{n}\right]_{0}\right| \leq C_{1}\left\|\left(\mathbf{e}^{n+1}, \mathbf{e}^{n}\right)\right\|_{E}, & n=1,2, \ldots, N-1, \\
\left\|\mathbf{e}^{n}-\left[\mathbf{e}^{n}\right]_{0}^{-} \mathbf{1}\right\| \leq C_{2}\left\|\left(\mathbf{e}^{n}, \mathbf{e}^{n-1}\right)\right\|_{E}, & n=1,2, \ldots, N-1,
\end{aligned}
$$

where $C_{1}, C_{2}$ are constants depending only on $r_{\max }$. On the other hand, in view of $(4.3)-(4.4)$,

$$
\begin{aligned}
\left\|\left(\mathbf{V}^{n}\right)^{2}-\left(\mathbf{W}^{n}\right)^{2}\right\| & \leq\left\|\left(\mathbf{V}^{n}+\mathbf{W}^{n}\right)\right\|_{\infty}\left\|\mathbf{e}^{n}\right\| \\
& \leq\left\{\left\|\mathbf{V}^{n}-\mathbf{u}^{n}\right\|_{\infty}+\left\|\mathbf{W}^{n}-\mathbf{u}^{n}\right\|_{\infty}+2\left\|\mathbf{u}^{n}\right\|_{\infty}\right\}\left\|\mathbf{e}^{n}\right\| \\
& \leq\left\{2 M+h^{-1 / 2}\left\|\mathbf{V}^{n}-\mathbf{u}^{n}\right\|+h^{-1 / 2}\left\|\mathbf{W}^{n}-\mathbf{u}^{n}\right\|\right\}\left\|\mathbf{e}^{n}\right\| \\
& \leq\left\{2 M+h^{-1 / 2} C_{2} \mu\right\}\left\|\mathbf{e}^{n}\right\|, \quad n=1,2, \ldots, N-1,
\end{aligned}
$$

where $C_{2}$ is another constant depending only on $r_{\max }$. On using these estimates in (4.8) we obtain

$$
\begin{array}{r}
\left(1-C_{1} k\right)\left\|\left(\mathbf{e}^{n+1}, \mathbf{e}^{n}\right)\right\|_{E} \leq\left(1+C_{4} k\right)\left\|\left(\mathbf{e}^{n}, \mathbf{e}^{n-1}\right)\right\|_{E}+\left\|D^{-2} \mathbf{e}^{n+1}\right\|, \\
n=1,2, \ldots, N-1,
\end{array}
$$


where $C_{4}$ depends on $\mu, M, r_{\max }$. The discrete Gronwall lemma now implies (4.5).

Of importance is the fact that the stability bound (4.5) does not hold for $\left\{\mathbf{V}^{n}\right\},\left\{\mathbf{W}^{n}\right\}$ arbitrary, but only for discrete solutions $\left\{\mathbf{V}^{n}\right\},\left\{\mathbf{W}^{n}\right\}$ near the theoretical solution ("near" in the sense that the threshold conditions (4.3), (4.4) hold). For the implications of this localized definition of stability, see $[7,8,3]$. For applications of the notion of stability threshold to other discretizations, see $[13,4,11]$.

We now turn to the study of the consistency of (2.8). The truncation errors $\mathscr{T}^{n}, n=0,1, \ldots, N$, are the residuals in the theoretical solution $\mathbf{u}^{n}$, defined as in (4.1) or (4.2):

$$
\begin{gathered}
\mathscr{T}^{0}=\mathbf{u}^{0}-\boldsymbol{\alpha}, \\
\mathscr{T}^{1}=\mathbf{u}^{1}-\boldsymbol{\alpha}-k \boldsymbol{\beta}, \\
\mathscr{T}^{n+1}=k^{-2}\left(\mathbf{u}^{n+1}-2 \mathbf{u}^{n}+\mathbf{u}^{n-1}\right)+\frac{1}{4}\left\{D^{4} \mathbf{u}^{n+1}+2 D^{4} \mathbf{u}^{n}+D^{4} \mathbf{u}^{n-1}\right\} \\
-D^{2} \mathbf{u}^{n}-D^{2}\left(\mathbf{u}^{n}\right)^{2}, \quad n=1,2, \ldots, N-1 .
\end{gathered}
$$

From (4.11) we get

$$
\begin{aligned}
D^{-2} \mathscr{T}^{n+1}= & D^{-2}\left[k^{-2}\left(\mathbf{u}^{n+1}-2 \mathbf{u}^{n}+\mathbf{u}^{n-1}\right)\right] \\
& +\frac{1}{4}\left\{D^{2} \mathbf{u}^{n+1}+2 D^{2} \mathbf{u}^{n}+D^{2} \mathbf{u}^{n-1}\right\} \\
& -\mathbf{u}^{n}+\left[\mathbf{u}^{n}\right]_{0}^{n} \mathbf{1}-\left(\mathbf{u}^{n}\right)^{2}\left[\left(\mathbf{u}^{n}\right)^{2}\right]_{0}^{-1}, \quad n=1,2, \ldots, N-1 .
\end{aligned}
$$

On the other hand, integration in (2.1) leads to

$$
0=-\partial_{x}^{-2} u_{t t}-\partial_{x}^{2} u+u-l(u)+u^{2}+l\left(u^{2}\right) \text {, }
$$

a result that when restricted to the grid and subtracted from (4.12) implies that $D^{-2} \mathscr{T}^{n+1}=\mathbf{T}_{1}^{n+1}+\mathbf{T}_{2}^{n+1}+\mathbf{T}_{3}^{n+1}+\mathbf{T}_{4}^{n+1}+\mathbf{T}_{5}^{n+1}+\mathbf{T}_{6}^{n+1}, \quad n=1,2, \ldots, N-1$, where, with $\mathbf{r}_{h}$ denoting, as before, grid restriction,

$$
\begin{aligned}
\mathbf{T}_{1}^{n+1} & =D^{-2}\left[k^{-2}\left(\mathbf{u}^{n+1}-2 \mathbf{u}^{n}+\mathbf{u}^{n-1}\right)-\mathbf{r}_{h} u_{t t}^{n}\right], \\
\mathbf{T}_{2}^{n+1} & =D^{-2} \mathbf{r}_{h} u_{t t}^{n}-\mathbf{r}_{h} \partial_{x}^{-2} u_{t t}^{n}, \\
\mathbf{T}_{3}^{n+1} & =\frac{1}{4}\left\{\left(D^{2} \mathbf{u}^{n+1}-\mathbf{r}_{h} \partial_{x}^{2} u^{n+1}\right)+2\left(D^{2} \mathbf{u}^{n}-\mathbf{r}_{h} \partial_{x}^{2} u^{n}\right)\right. \\
& \left.+\left(D^{2} \mathbf{u}^{n-1}-\mathbf{r}_{h} \partial_{x}^{2} u^{n-1}\right)\right\}, \\
\mathbf{T}_{4}^{n+1} & =\frac{1}{4} \mathbf{r}_{h}\left(\partial_{x}^{2} u^{n+1}+2 \partial_{x}^{2} u^{n}+\partial_{x}^{2} u^{n-1}-4 \partial_{x}^{2} u^{n}\right), \\
\mathbf{T}_{5}^{n+1} & =\left(-\left[\mathbf{u}^{n}\right]_{0}^{\hat{n}}+l\left(u^{n}\right)\right) \mathbf{1}, \\
\mathbf{T}_{6}^{n+1} & =\left(-\left[\left(\mathbf{u}^{n}\right)^{2}\right]_{0}^{\hat{+}}+l\left(\left(u^{n}\right)^{2}\right)\right) \mathbf{1} .
\end{aligned}
$$

For the first and fourth term, which are linked to the discretization in time, we can write $\max _{n}\left\|\mathbf{T}_{1}^{n+1}\right\|=\mathscr{O}\left(k^{2}\right), \max _{n}\left\|\mathbf{T}_{4}^{n+1}\right\|=\mathscr{O}\left(k^{2}\right)$ if, say, $u_{x x t t}$ and $u_{t t t t}$ 
are bounded in $0 \leq x \leq 1,0 \leq t \leq T$. The remaining terms $\mathbf{T}_{2}^{n+1}, \mathbf{T}_{3}^{n+1}$, $\mathbf{T}_{5}^{n+1}, \mathbf{T}_{6}^{n+1}$ come from the discretization in space. It is in these terms that the accuracy of the pseudospectral technique should manifest itself. For instance, by reasoning as in the proof of Lemma 2.2 in [14], it is easily shown that, if $u_{t t}\left(\cdot, t_{n}\right)$ belongs to the periodic Sobolev space $H^{s}, s>1 / 2$, then $\left\|\mathbf{T}_{2}^{n+1}\right\| \leq$ $C_{s} h^{s}\left\|u_{t t}\left(\cdot, t_{n}\right)\right\|_{H^{s}}$. Thus, for $u_{t t}$ sufficiently smooth, $\left\|\mathbf{T}_{2}^{n+1}\right\|=\mathscr{O}\left(h^{s}\right)$, for any $s>0$ (in fact, this term may even be exponentially small, see [14]). In a similar way, $\max _{n}\left\|\mathbf{T}_{3}^{n+1}\right\|$ is $\mathscr{O}\left(h^{s}\right)$, if $u\left(\cdot, t_{n}\right)$ is in $H^{s+2}$ uniformly in $t$ [14, Lemma 2.2]. Finally, the aliasing errors $\left\|\mathbf{T}_{5}^{n+1}\right\|,\left\|\mathbf{T}_{6}^{n+1}\right\|$ are $\mathscr{O}\left(h^{s}\right)$ if $u, u^{2}$ respectively are in $H^{s}$, uniformly in $t$. To sum up, for the truncation error it is possible to derive bounds

$$
\max _{1 \leq n \leq N-1}\left\|D^{-2} \mathscr{T}^{n+1}\right\|=\mathscr{O}\left(k^{2}+h^{s}\right), \quad h, k \rightarrow 0,
$$

provided that the theoretical solution $u$ is sufficiently smooth. Furthermore, it would also be possible to replace the term $h^{s}$ by an exponential term $\exp (-1 / h)$ under further smoothness assumptions.

We now investigate the behavior of the truncation errors $\mathscr{T}^{0}, \mathscr{T}^{1}$ associated with the starting values of the algorithm. In view of the structure of the right-hand side of $(4.5), \mathscr{T}^{0}, \mathscr{T}^{1}$ contribute to the bound for the global error through $\left\|\left(\mathscr{T}^{1}, \mathscr{T}^{0}\right)\right\|_{E}$. Therefore, we should require

$$
\left\|\left(\mathscr{T}^{1}, \mathscr{T}^{0}\right)\right\|_{E}=\mathscr{O}\left(k^{2}+h^{s}\right), \quad h, k \rightarrow 0,
$$

if the error in approximating the initial conditions is not to dominate over the truncation error of (2.8). Note that, by (3.7), the estimate (4.14) holds if $\alpha$ is chosen to be the restriction $\mathbf{u}^{0}$ of $u^{0}$ and $\boldsymbol{\beta}$ satisfies

$$
\left\|\left(\mathbf{u}^{1}-\mathbf{u}^{0}\right) / k-\boldsymbol{\beta}\right\|_{E}=\mathscr{O}\left(k^{2}+h^{s}\right), \quad h, k \rightarrow 0 .
$$

The relation (4.15), in turn, holds if $u$ is smooth and $\boldsymbol{\beta}$ is taken to be the grid restriction of the following Taylor expansion of $u_{t}(\cdot, k / 2)$ :

$$
\left.\left(u_{t}+(k / 2) u_{t t}\right)\right|_{t=0} \text {. }
$$

Note that $u_{t t}(t=0)$ is available from differentiation of $u^{0}$ with respect to $x$ in the differential equation (2.1). Alternatively, the $x$-derivatives of $u^{0}$ required can be replaced by pseudospectral differences.

We are now in a position to prove the convergence of the method.

Theorem 4.2. Suppose that the hypotheses of the stability theorem 4.1 hold. Assume that the choice of starting vectors $\boldsymbol{\alpha}, \boldsymbol{\beta}$ and the smoothness of the theoretical solution guarantee that (4.13)-(4.14) hold with $s>1 / 2$. Then

$$
\max _{1 \leq n \leq N-1}\left\|\mathbf{U}^{n+1}-\mathbf{u}^{n+1}, \mathbf{U}^{n}-\mathbf{u}^{n}\right\|_{E}=\mathscr{O}\left(k^{2}+h^{s}\right), \quad h, k \rightarrow 0
$$

so that in particular the following $L^{2}$-estimate holds:

$$
\max _{0 \leq n \leq N}\left\|\mathbf{U}^{n}-\mathbf{u}^{n}\right\|=\mathscr{O}\left(k^{2}+h^{s}\right), \quad h, k \rightarrow 0 .
$$


Proof. Basically, we apply the stability bound with the choice $\mathbf{V}^{n}=\mathbf{U}^{n}, \mathbf{W}^{n}=$ $\mathbf{u}^{n}, n=0,1, \ldots, N$. However, it is not clear that, with this choice, the threshold conditions (4.4) hold. It is then necessary to apply the main theorem of [7], based on an important lemma due to Stetter [12, Lemma 1.2.1].

It is of course clear that, for $u$ sufficiently smooth, the estimates $\mathscr{O}\left(h^{s}\right)$ for the spatial contribution can be replaced by exponential estimates $\mathscr{O}(\exp (-1 / h))$.

Remark. As a first step in the analysis in this section, the differential equation and the numerical method have been integrated twice with respect to $x$. This device was introduced to alleviate the difficulties found in the stability analysis of the nonlinear term: dealing with $\left(\mathbf{V}^{n}\right)^{2}-\left(\mathbf{W}^{n}\right)^{2}$ is easier than dealing with $D^{2}\left(\mathbf{V}^{n}\right)^{2}-D^{2}\left(\mathbf{W}^{n}\right)^{2}$. As a result, our energy norm is an $L^{2}$-norm of $u$ combined with a negative norm of $u_{t}$. This should be compared with the energy norm in [8]: there, no integration with respect to $x$ is necessary and convergence is proved in $H_{2}$ for $u$ and $L_{2}$ for $u_{t}$.

\section{NUMERICAL EXPERIMENTS}

The pseudospectral scheme analyzed above has been tested in the long-time integration of solitary waves and collisions of solitary waves.

The solitary wave of the GB equation is given by

$$
u(x, t)=-A \operatorname{sech}^{2}\left[(P / 2)\left(\xi-\xi_{0}\right)\right], \quad \xi=x-c t,
$$

where $\xi_{0}$ and $P$ are real parameters, $0<P \leq 1$, and the amplitude $A$ and velocity $c$ of the wave are related to $P$ through the formulas

$$
A=3 P^{2} / 2, \quad c= \pm\left(1-P^{2}\right)^{1 / 2} .
$$

Equation (5.1) shows that the solitary waves decay exponentially as $|x| \rightarrow \infty$. Therefore, it is possible to use the periodic pseudospectral scheme (2.8) on an interval $\left[x_{L}, x_{R}\right]$, where the artificial boundaries are located far out enough for the theoretical solution to satisfy the conditions

$$
\left(\partial^{i} / \partial x^{i}\right) u\left(x_{L}, t\right)=\left(\partial^{i} / \partial x^{i}\right) u\left(x_{R}, t\right), \quad 0 \leq t \leq T, i=0,1,2,3,
$$

except for a negligible remainder.

The scheme was implemented in single precision on a VAX-11/780 machine with a VAX-11 FORTRAN compiler. The Fourier transforms were carried out by the Cooley-Tuckey [2] algorithm coded by us in FORTRAN.

TABLE 1

Single soliton error

\begin{tabular}{|rlllll|}
\hline $2 J$ & $h$ & $k=0.8$ & $k=0.4$ & $k=0.2$ & $k=0.1$ \\
\hline 32 & 2.5 & 0.0805 & 0.0729 & 0.0717 & 0.0715 \\
64 & 1.25 & 0.0328 & 0.0078 & 0.0019 & 0.0005 \\
128 & 0.625 & unstable & 0.0078 & 0.0019 & 0.0005 \\
\hline
\end{tabular}


Table 1 corresponds to a single soliton (5.1) with a (moderate) amplitude $A=0.5$ and an initial phase $\xi_{0}=0$. The missing starting level $\mathbf{U}^{1}$ was obtained as in (4.16), boundaries were placed at $x_{L}=-40, x_{R}=40$, and the integration was followed up to $T=40$. The table provides the $L^{2}$-errors at the final time.

Note that, for $2 J=32$, a reduction in $k$ does not significantly change the error. This shows that for this value of $J$ the error orginates, almost entirely, from the space discretization (i.e., the results given by the scheme are the same as those of the time-continuous version of the pseudospectral method). However, when $2 J$ is doubled to $2 J=64$, the picture is different: halving $k$ results in a division by 4 of the error, showing that now the space discretization is far more accurate than the integration in time. This is confirmed by the fact that a further doubling of $2 \mathrm{~J}$ from 64 to 128 has no effect on the error for $k \geq 0.4$. For $2 J=128, k=0.8$, we have observed marked solution growth. This does not contradict the stability theorem 4.1, since that result holds only for $k$ small enough with respect to the mesh ratio. Observe that in this and later experiments the unstable runs correspond to situations where a large value of $k$ is combined with a large value of $r$. Before leaving Table 1, we would like to emphasize that the great gain in accuracy observed when comparing the two runs $2 J=32, k=0.1$ and $2 J=64, k=0.1$ nicely illustrates the consistency advantages of the spectral technique.

TABLE 2

Collision errors/pseudospectral

\begin{tabular}{|ccccc|}
\hline $2 J$ & $h$ & $k=0.8$ & $k=0.4$ & $k=0.2$ \\
\hline 32 & 3.75 & 0.0848 & 0.0821 & 0.0816 \\
& & $(0.8)$ & $(1.8)$ & $(3.6)$ \\
64 & 1.875 & $\begin{array}{l}0.0053 \\
(1.7)\end{array}$ & $\begin{array}{c}0.0014 \\
(3.3)\end{array}$ & $\begin{array}{l}0.0005 \\
(6.6)\end{array}$ \\
\hline
\end{tabular}

Table 2 corresponds to a collision of two solitons of equal (small) amplitude $A_{1}=A_{2}=0.25$, initially located at $x=-20$ and $x=20$, respectively. Since the amplitudes are small, the solitons emerge from the interaction without changes in shape or velocity. The theoretical solution is given by a rather complicated expression that can be seen in [9] or [10]. Again, $T=40$, but now $x_{L}=60, x_{R}=60$, and $\mathrm{U}^{1}$ was taken from the theoretical solutions. The table provides the $L^{2}$-errors at the final time, along with the corresponding CPU times in seconds (quantities in parentheses). The spectral accuracy property is again clearly visible and, in fact, the errors for $2 J=128$ (not given in the table) are identical to those for $2 J=64$. 
In order to gain some appreciation of the performance of algorithms based on spatial finite differences, we have implemented the scheme

$$
\begin{aligned}
k^{-2}\left(\mathbf{U}^{n+1}-2 \mathbf{U}^{n}+\mathbf{U}^{n-1}\right)= & -\frac{1}{4}\left\{D^{*} \mathbf{U}^{n+1}+2 D^{* 4} \mathbf{U}^{n}+\mathbf{D}^{* 4} \mathbf{U}^{n-1}\right\} \\
& +D^{* 2} \mathbf{U}^{n}+D^{* 2}\left(\mathbf{U}^{n}\right)^{2},
\end{aligned}
$$

where $D^{* 2}, D^{* 4}$ are the standard central difference discretizations of $\partial^{2} / \partial x^{2}$, $\partial^{4} / \partial x^{4}$, respectively. Note that $(2.8)$ and (5.2) are identical as far as the timeintegration is concerned. The computation of $\mathbf{U}^{n+1}$ in (5.2) requires the solution of a linear system whose matrix can be factored once and for all at the beginning of the time-stepping. To simplify the structure of this matrix, (5.2) was implemented with the boundary conditions $u=u_{x}=0$ at $x=x_{L}, x_{R}$, rather than with periodicity conditions. This is a standard practice in this sort of problem (cf., e.g., the experiments in [11]). The method (5.2) is clearly second-order accurate in space and time. Furthermore, it can be shown to be stable in the sense of Theorem 4.1.

TABLE 3

Collision errors/finite differences

\begin{tabular}{|ccccc|}
\hline $2 J$ & $h$ & $k=0.8$ & $k=0.4$ & $k=0.2$ \\
\hline 128 & 0.9375 & 0.0407 & 0.0401 & 0.0409 \\
& & $(0.6)$ & $(1.1)$ & $(2.3)$ \\
256 & 0.46875 & unstable & 0.0092 & 0.0092 \\
& & & $(4.1 * * *)$ & $(8.2 * * *)$ \\
\hline
\end{tabular}

Table 3 corresponds to the application of (5.2) to the collision experiment that was integrated before by the pseudospectral method. Here we give results for $2 J=128,256$, as those for $2 J=32,64$ are very inaccurate. Note the $\mathscr{O}\left(h^{2}\right)$ behavior of the error. The stars on the CPU times mean that the run was carried out in double precision, since the single-precision results were badly affected by roundoff errors. A comparison of the errors in Tables 2 and 3 shows that for given $h$ and $k$, the pseudospectral scheme is definitely more accurate than its finite difference counterpart. When computer times are taken into account, finite differences do well if low accuracy is required (for instance, they can give errors below 0.05 with 0.6 seconds of CPU). However, if smaller errors are required (say below 0.005 ), the pseudospectral method should be preferred. It should also be kept in mind that a better coding of the FFT would further enhance the efficiency of the pseudospectral algorithm.

\section{ACKNOWLEDGMENT}

This work is part of the Project PB-86-0313 supported by "Fondo Nacional para el desarrollo de la Investigación Cientifica y Técnica.” 


\section{BIBLIOGRAPHY}

1. C. Canuto, M. Y. Hussaini, A. Quarteroni, and T. A. Zang, Spectral methods in fluid dynamics, Springer-Verlag, New York, 1988.

2. J. W. Cooley and J. W. Tukey, An algorithm for the machine calculation of complex Fourier series, Math. Comp. 19 (1965), 297-301.

3. J. de Frutos and J. M. Sanz-Serna, h-dependent stability thresholds avoid the need for a priori bounds in nonlinear convergence proofs, Computational Mathematics III, Proc. Third Internat. Conf. held in Benin City, Nigeria, January 1988 (S. O. Fatunla, ed.), Boole Press, Dublin (to appear).

4. __ Split-step spectral schemes for nonlinear Dirac systems, J. Comput. Phys. 83 (1989), 407-423.

5. B. Fornberg, On a Fourier method for the integration of hyperbolic equations, SIAM J. Numer. Anal. 12 (1975), 509-528.

6. D. Gottlieb and S. A. Orszag, Numerical analysis of spectral methods: Theory and applications, SIAM, Philadelphia, PA, 1977.

7. J. C. López-Marcos and J. M. Sanz-Serna, A definition of stability for nonlinear problems, Numerical Treatment of Differential Equations, Proc. Fourth Seminar "NUMDIFF-4" held in Halle 1987 (K. Strehmel, ed.), Teubner-Texte zur Mathematik, Leipzig, 1988, pp. 216226.

8. __ Stability and convergence in numerical analysis. III: Linear investigation of nonlinear stability, IMA J. Numer. Anal. 7 (1988) 71-84.

9. V. S. Manoranjan, A. R. Mitchell, and J. LL. Morris, Numerical solution of the "good" Boussinesq equation, SIAM J. Sci. Statist. Comput. 5 (1984), 946-957.

10. V. S. Manoranjan, T. Ortega, and J. M. Sanz-Serna, Soliton and anti-soliton interactions in the "good" Boussinesq equation, J. Math. Phys. 29 (1988), 1964-1968.

11. T. Ortega and J. M. Sanz-Serna, Nonlinear stability and convergence of finite-difference methods for the "good" Boussinesq equation, Numer. Math. 58 (1990), 215-229.

12. H. J. Stetter, Analysis of discretization methods for ordinary differential equations, Springer, Berlin, 1973.

13. E. Suli, Convergence and nonlinear stability of the Lagrange-Galerkin method for the NavierStokes equation, Numer. Math. 53 (1988), 459-484.

14. E. Tadmor, The exponential accuracy of Fourier and Chebyshev differencing methods, SIAM J. Numer. Anal. 23 (1986), 1-10.

15. T. R. Taha and M. J. Ablowitz, Analytical and numerical aspects of certain nonlinear evolution equation. II: Numerical nonlinear Schrödinger equation, J. Comput. Phys. 55 (1984), 203-230.

16. R. G. Voigt, D. Gottlieb, and M. Y. Hussaini (eds.), Spectral methods for partial differential equations, SIAM, Philadelphia, PA, 1984.

17. J. A. C. Weideman and B. M. Herbst, Split-step methods for the solution of the nonlinear Schrödinger equation, SIAM J. Numer. Anal. 23 (1986), 485-507.

Departamento de Matemática Aplicada y Computación, Facultad de Ciencias, UniVersidad de Valladolid, Valladolid, Spain 